\title{
A simple shock-capturing technique for high-order discontinuous Galerkin methods
}

\author{
A. Huerta ${ }^{1, *, \dagger}$, E. Casoni ${ }^{1}$ and J. Peraire ${ }^{2}$ \\ ${ }^{1}$ Laboratori de Calcul Numeric, Departament de Matematica Aplicada III, E.T.S. de Ingenieros de Caminos, \\ Canales y Puertos, Universitat Politecnica de Catalunya, Jordi Girona 1-3, 08034 Barcelona, Spain \\ ${ }^{2}$ Department of Aeronautics and Astronautics, Massachusetts Institute of Technology, Cambridge, MA 02139, USA
}

\begin{abstract}
SUMMARY
This article presents a novel shock-capturing technique for the discontinuous Galerkin (DG) method. The technique is designed for compressible flow problems, which are usually characterized by the presence of strong shocks and discontinuities. The inherent structure of standard DG methods seems to suggest that they are especially adapted to capture shocks because of the numerical fluxes based on suitable approximate Riemann solvers, which, in practice, introduces some stabilization. However, the usual numerical fluxes are not sufficient to stabilize the solution in the presence of shocks for large high-order elements. Here, a new basis of shape functions is introduced. It has the ability to change locally between a continuous or discontinuous interpolation depending on the smoothness of the approximated function. In the presence of shocks, the new discontinuities inside an element introduce the required stabilization because of numerical fluxes. Large high-order elements can therefore be used and shocks captured within a single element, avoiding adaptive mesh refinement and preserving the locality and compactness of the DG scheme. Several numerical examples for transonic and supersonic flows are studied to demonstrate the applicability of the proposed approach.
\end{abstract}

KEY WORDS: Shock-capturing; discontinuous Galerkin; high order; Euler equations; compressible flow; Euler flow; discontinous enrichment; adaptivity

\section{INTRODUCTION}

In the last decades, discontinuous Galerkin (DG) methods [1-8] have focused many studies on nonlinear conservation laws and convection-dominated problems. Some CFD applications may require highly accurate numerical models to obtain high-fidelity predictions. DG methods are a good alternative for the solution of the Euler equations of gas dynamics. These methods combine in an optimal manner the high-order discretization tools of the general Galerkin finite elements and the local conservation properties typical of finite volume (FV) technology, which has been successful in the simulation of flows with discontinuities $[9,10]$. Moreover, DG methods are well-suited for highorder $p$-adaptive discretizations on structured and unstructured meshes, providing also a compact scheme.

This paper focuses on shock-capturing for high-order $(p \geqslant 2) \mathrm{DG}$ approximations. The inherent stability of DG methods produces adequate solutions but only for constant or linear approximations and fine enough discretizations. Note however, that standard high-order DG approximations require further stabilization. Moreover, as in many other methods, high-order discretizations produce oscillations when the approximating space is inadequate to resolve the main features of the true solution,

*Correspondence to: A. Huerta, Laboratori de Càlcul Numèric (LaCàN), E.T.S. Ingenieros de Caminos, Universitat Politècnica de Catalunya, Jordi Girona 1, E-08034 Barcelona, Spain.

†E-mail: antonio.huerta@upc.es 
such as unresolved shocks. To overcome this drawback many researchers have extended classical shock-capturing methodologies of finite differences and FV schemes to high-order DG methods.

The key in shock-capturing schemes is dissipation. In fact, artificial diffusion was already proposed in the 1950s by von Neumann and Richtmyer [11]. It consists of explicitly adding viscous terms to the governing partial differential equation. The idea behind adding artificial viscosity is to spread the discontinuity over a length scale so that it can be resolved in the space of interpolating functions. Persson and Peraire [12] introduced a $p$-dependent artificial viscosity that scales like $h / p$, hence obtaining a shock width $\delta \simeq C h / p$. Recently, some authors have also recovered the artificial diffusion technique, obtaining subcell resolution (see, e.g. [13, 14]). In [15], the same authors proposed an artificial diffusion of order $\mathcal{O}\left(h^{k}\right)$ with $1 \leqslant k \leqslant p$, which for $p$ high enough improves the threshold $h / p$. All these approaches require a robust discontinuity detection procedure to properly locate the shock (see, e.g. [12]). Although artificial diffusion techniques provide a robust and accurate approach to capture shocks with high-order DG methods, computing artificial viscosity is not trivial because of its nonlinearity and the difficulty of incorporating directionality. In addition, the introduction of a viscous term in the original equation implies treating high-order derivatives in the PDE.

Another successful approach for shock capturing are limiting techniques. These methods were initially designed in the context of finite differences and FV [16] and later applied to DG methods. See, for instance, the classical Runge-Kutta discontinuous Galerkin methods [2,3,17]. They combine approximate Riemann solvers and nonlinear operators (i.e. slope limiters) to satisfy total variation bounded in the means. Although well behaved limiters for second-order schemes have been developed, the extension to higher order approximations is still open. While these limiters have been carefully designed to avoid oscillations, they drastically reduce the order of the approx-
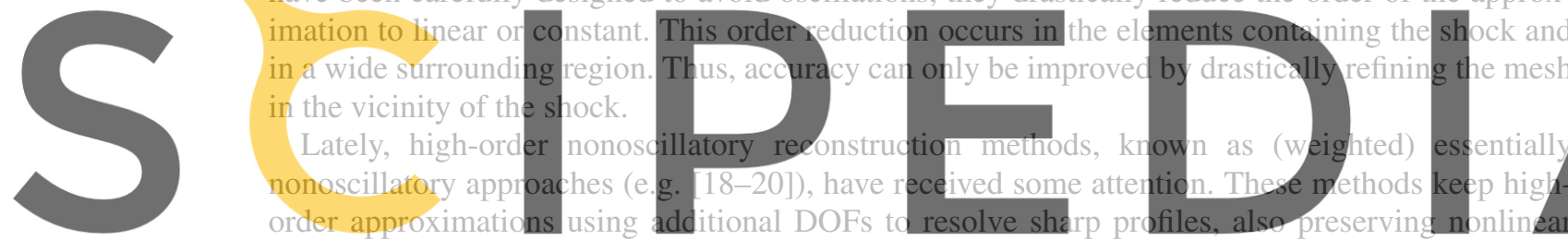

stability. However, their computational overhead is excessive for high-order approximations. They

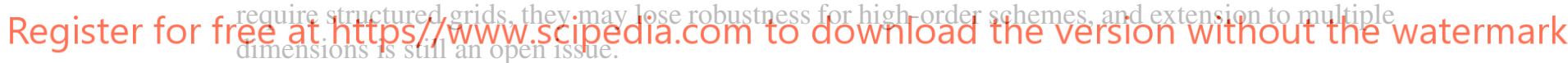

In this paper, a simple strategy, exploiting the stability introduced by numerical fluxes, is presented. Basis functions may vary from piecewise constant to a continuous representation of the solution within each element. By means of a shock capturing technique, the order of the approximation is reduced to one only in the elements where the solution is not smooth. Thus, high-accuracy of order $h^{p}$ in the large majority of the domain, which is locally decreased to order $h / p$ only in the elements where the shock is contained. Mesh adaption is thus not mandatory and shocks may be captured without modifying the number of DOFs or the mesh topology. In fact, an inherent adaption both in time and space is designed to work with large high-order elements.

The outline of the paper is as follows. In Section 2 the formulation of the standard DG method for the compressible Euler equations of gas dynamics is briefly reviewed. Section 3 discusses the details regarding the basis of shape functions introduced, with special attention to the construction of the constant shape functions, the control of the discontinuities inside an element and the discontinuity detection mechanism. In Section 4 numerical examples show the applicability of this approach to unsteady and steady problems and demonstrate the efficiency of the method for high-order approximations.

\section{THE DISCONTINUOUS GALERKIN METHOD FOR THE EULER EQUATIONS}

This section recalls basic concepts of the DG method for the solution of Euler equations. These equations impose conservation of mass, momentum and energy for a compressible, inviscid and nonconducting fluid. The strong form of these conservation laws in the absence of external volume 
forces, can be expressed in a bounded domain $\Omega \subset \mathbb{R}^{n_{\mathrm{sd}}}$, with $n_{\mathrm{sd}}$ the number of spatial dimensions, as

$$
\frac{\partial \mathbf{U}}{\partial t}+\frac{\partial \mathbf{F}_{k}(\mathbf{U})}{\partial x_{k}}=\mathbf{0}
$$

where $k$ ranges from 1 to $n_{\text {sd }}, \mathbf{U}$ is the vector of conservation variables, and $\mathbf{F}_{k}(\mathbf{U})$ is the flux vector for each spatial dimension $x_{k}$. Obviously, appropriate boundary conditions must be imposed on $\partial \Omega$ (see, e.g. [21]). In particular, for $n_{\text {sd }}=2$, which is the case studied here, the vectors in (1) are

$$
\mathbf{U}=\left(\begin{array}{c}
\rho \\
\rho v_{1} \\
\rho v_{2} \\
\rho E
\end{array}\right) \quad \mathbf{F}_{1}=\left(\begin{array}{c}
\rho v_{1} \\
\rho v_{1}^{2}+\mathrm{p} \\
\rho v_{1} v_{2} \\
v_{1}(\rho E+\mathrm{p})
\end{array}\right) \quad \text { and } \quad \mathbf{F}_{2}=\left(\begin{array}{c}
\rho v_{2} \\
\rho v_{1} v_{2} \\
\rho v_{2}^{2}+p \\
v_{2}(\rho E+p)
\end{array}\right)
$$

where $\rho$ is density, $\boldsymbol{v}=\left(v_{1}, v_{2}\right)^{T}$ is fluid velocity, $E$ is energy and $p$ is pressure.

An equation of state, relating the internal energy to pressure and density, is needed to close the system of nonlinear hyperbolic equations. For a perfect polytropic gas, the equation of state is $p=(\gamma-1) \rho\left(E-(1 / 2)\|v\|^{2}\right)$ with $\gamma=1.4$ for air. Finally, it is important to recall the definition of the Mach number $M=\|v\| / c$, where $c=\sqrt{\gamma \mathrm{p} / \rho}$ is the speed of sound.

To apply the DG method, the computational domain $\Omega$ is partitioned in $n_{\mathrm{el}}$ disjoint subdomains with a characteristic size $h$, that is
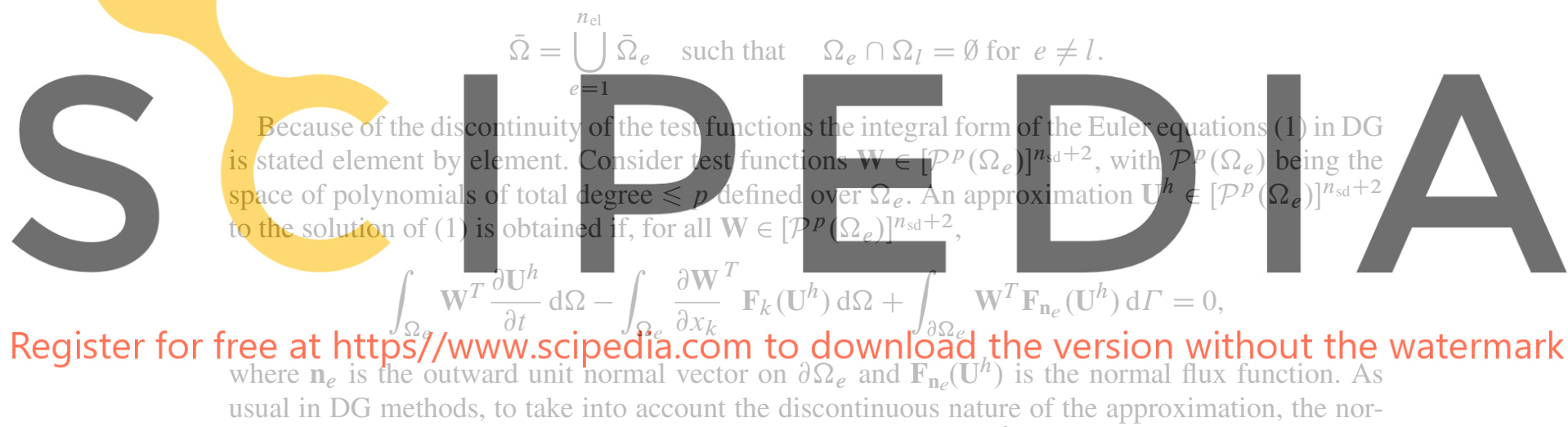

mal flux at the element boundary is replaced by a numerical one, $\hat{\mathbf{F}}_{\mathbf{n}_{e}}\left(\mathbf{U}^{h}, \mathbf{U}_{\text {out }}^{h}\right)$, which is evaluated in terms of the solution in the current element $\Omega_{e}, \mathbf{U}^{h}$, and the solution at neighboring elements,

$$
\mathbf{U}_{\text {out }}^{h}=\lim _{\varepsilon \rightarrow 0^{+}} \mathbf{U}^{h}\left(\boldsymbol{x}+\varepsilon \mathbf{n}_{e}\right) \quad \text { for } \quad \boldsymbol{x} \in \partial \Omega_{e} .
$$

This interface flux function, $\hat{\mathbf{F}}_{\mathbf{n}_{e}}$ is determined using any standard Riemann solver ([10]). Here, a flux function of the Roe type is selected [22]. Hence, the resulting DG formulation for element $\Omega_{e}$ $\left(e=1, \ldots, n_{\mathrm{el}}\right)$ becomes find $\mathbf{U}^{h} \in\left[\mathcal{P}^{p}\left(\Omega_{e}\right)\right]^{n_{\mathrm{sd}}+2}$ such that, for all $\mathbf{W} \in\left[\mathcal{P}^{p}\left(\Omega_{e}\right)\right]^{n_{\mathrm{sd}}+2}$,

$$
\int_{\Omega_{e}} \mathbf{W}^{T} \frac{\partial \mathbf{U}^{h}}{\partial t} \mathrm{~d} \Omega-\int_{\Omega_{e}} \frac{\partial \mathbf{W}^{T}}{\partial x_{k}} \mathbf{F}_{k}\left(\mathbf{U}^{h}\right) \mathrm{d} \Omega+\int_{\partial \Omega_{e}} \mathbf{W}^{T} \hat{\mathbf{F}}_{\mathbf{n}_{e}}\left(\mathbf{U}, \mathbf{U}_{\text {out }}^{h}\right) \mathrm{d} \Gamma=0 .
$$

This induces a system of ODEs that can be solved with any standard time integration scheme. Here, an explicit Runge-Kutta method is used [21]. The time-step is determined by the stability condition, which limits the Courant number as follows

$$
C=\left|\lambda_{\max }\right| \frac{\Delta t}{h} \leqslant \frac{1}{2 p+1},
$$

where $h$ is the element size and $p$ the degree of the functional approximation. The scalar value $\lambda_{\max }$ is the maximum eigenvalue of the Jacobian matrix of the flux. Recall that for a system of hyperbolic conservation laws, the eigenvalues $\lambda_{j}$, for $j=1, \ldots, n_{\mathrm{sd}}+2$, correspond to the velocities of the characteristic quantities (for more details see [21,23]). 


\section{DEFINITION OF THE APPROXIMATION SPACE}

\subsection{From continuous to discontinuous}

The solution $\mathbf{U}^{h}$ of Equation (2) is sought in $\left[\mathcal{P}^{p}\left(\Omega_{e}\right)\right]^{n_{\mathrm{sd}}+2}$, which is characterized by a complete basis of polynomials; that is,

$$
\mathcal{P}^{p}\left(\Omega_{e}\right)=\operatorname{span}\left\{N_{1}(\boldsymbol{x}), N_{2}(\boldsymbol{x}), \ldots, N_{n_{\mathrm{en}}}(p)(\boldsymbol{x})\right\} \quad \text { for } \boldsymbol{x} \in \Omega_{e}
$$

where $n_{\mathrm{en}}(p)$ is the number of element nodes (i.e. the number of DOFs for each element), which depends on the degree $p$ of the basis.

Here, the polynomial space is modified to capture discontinuities. The new shape functions, $\bar{N}_{i}(\boldsymbol{x} ; \alpha)$, are defined as a convex combination of standard polynomials, $N_{i}(\boldsymbol{x})$, and a set of piecewise constant functions within each subcell of the element, $\phi_{i}(\boldsymbol{x})$. That is, the approximation is now characterized by

$$
\mathbb{U}^{h}=\sum_{i=1}^{n_{\mathrm{en}}(p)} \bar{N}_{i}(x ; \alpha) \mathbb{U}_{i}^{h} \quad \text { for } x \in \Omega_{e},
$$

and the shape functions are defined as follows:

$$
\bar{N}_{i}(x ; \alpha):=\alpha N_{i}(x)+(1-\alpha) \phi_{i}(x) \text { for } i=1, \ldots, n_{\mathrm{en}}(p)
$$

where $\alpha \in[0,1]$ is a parameter that depends on the smoothness of the solution.

Each element $\Omega_{e}$ is arbitrarily partitioned into a set of $n_{\mathrm{en}}(p)$ nonoverlapping subcells to
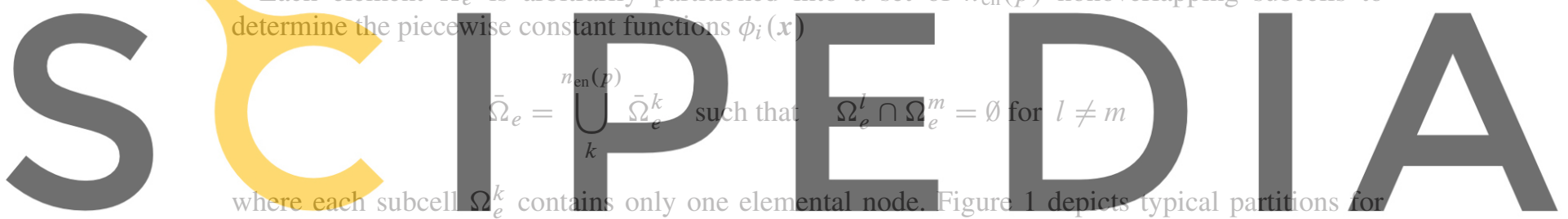

a first-order and second-order element. Notice that the cell interfaces form a nonstructured mesh.

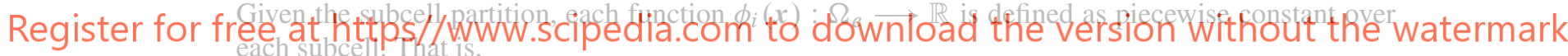

$$
\forall x \in \Omega_{e} \quad \phi_{i}(x)=\phi_{i}^{k}=\mathrm{cst} \quad \text { if } x \in \Omega_{e}^{k}, \quad \text { for } k=1, \ldots, n_{\mathrm{en}}(p)
$$

where $\phi_{i}^{k}$ are defined in the next subsection.

Remark 1

The partition of $\Omega_{e}$ into control volumes is not unique. Here, a simple procedure is considered: given a triangulation of the element, obtained by joining its nodes, the centroid of each subtriangle and the midpoints of the edges form a set of $n_{\mathrm{en}}(p)$ polygons such that each of them contains only one elemental node.
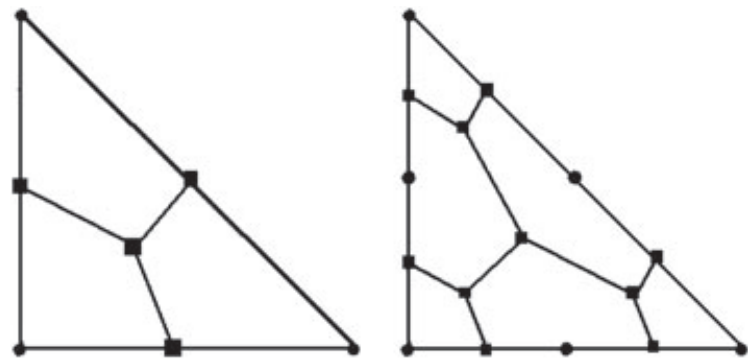

Figure 1. Cell partition of $\Omega_{e}, p=1$ (left) and $p=2$ (right). Circle marks correspond to node positions and square marks are the subcell vertices. 
A major advantage of DG methods is the intrinsic stabilization introduced by numerical fluxes, see for instance [5,24]. For values of $\alpha \in[0,1$ [ the proposed basis of approximation functions, namely

$$
\operatorname{span}\left\{\bar{N}_{1}(x ; \alpha), \bar{N}_{2}(x ; \alpha), \ldots, \bar{N}_{n_{\mathrm{en}}(p)}(x ; \alpha)\right\} \text { for } x \in \Omega_{e},
$$

introduces interelement discontinuities because of the piecewise constant contribution of each $\phi_{i}(x)$. Thus, numerical fluxes are accounted for inside the elements and their influence on stability will preclude spurious oscillations. Obviously, the order of the approximation is reduced to one in the element when $\alpha \in[0,1[$ and, as described below, the shock resolution is $\mathcal{O}(h / p)$. Whereas high-accuracy, of order $h^{p}$, is retained in the elements with $\alpha=1$, which define the large majority of the domain as it will be shown in the examples.

Figure 2 shows a typical third-order shape function $\bar{N}_{i}$ for different values of $\alpha$. The particular cases $\bar{N}_{i}=\phi_{i}$ and $\bar{N}_{i}=N_{i}$ correspond to Figures 2(a) and (b), respectively.

Parameter $\alpha$ can vary in space and time depending on the regularity of the solution. Consequently, although the number of DOFs and the topology of the mesh is unchanged the interpolation is adapted (in space and time) to the smoothness of the solution by means of $\alpha$ and its influence in the approximation basis (see Equation (3)).

\subsection{Piecewise constant subcell shape functions}

In the limit case of $\alpha=0$ the discontinuous functions $\phi_{i}$ induce a standard finite volume approach over the subcells $[10,25,26]$. Thus, conservation is also imposed for each subcell for any value of $\alpha$. Thus, to design functions $\phi_{i}$ standard zero-order reproducibility is imposed. Namely,
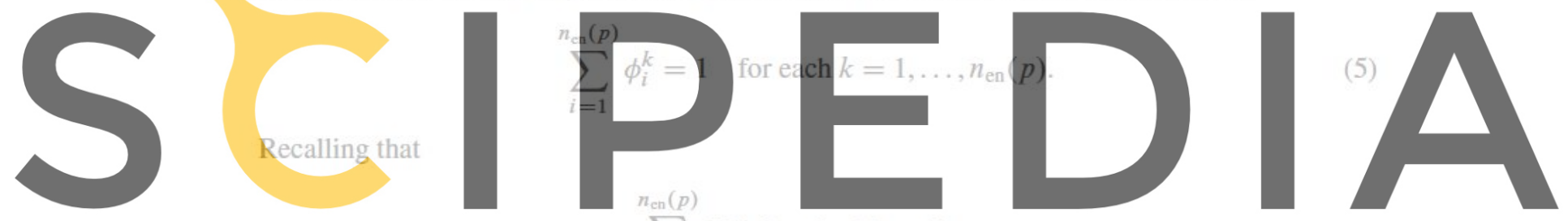

Register for free at https//www.scipedia.'com to download the version without the watermark Equation (5) implies

$$
\sum_{i=1}^{n_{\mathrm{cn}}(p)} \bar{N}_{i}(x ; \alpha)=1 \quad \forall x \in \Omega_{e} \text { and } \forall \alpha \in[0,1] .
$$

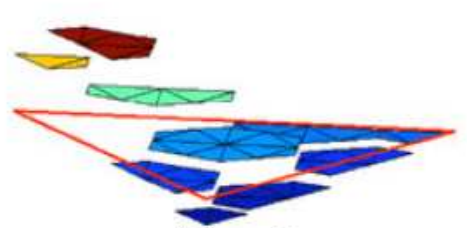

(a) $\alpha=0$

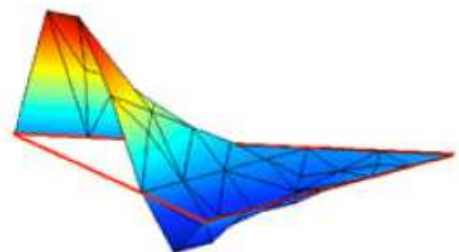

(b) $\alpha=1$

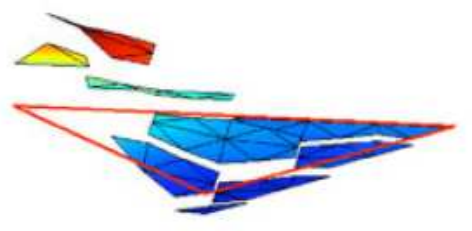

(c) $\alpha=0.25$

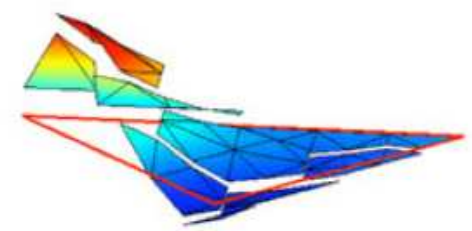

(d) $\alpha=0.5$

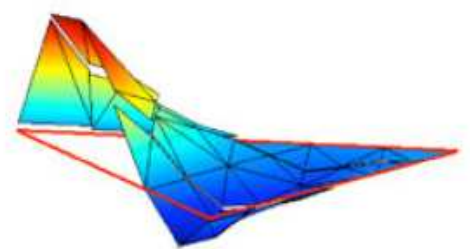

(e) $\alpha=0.85$

Figure 2. Shape function of degree $p=3$ for different values of $\alpha$. 
There are several options to define $\phi_{i}^{k}$ to satisfy Equation (5), but it is important to recall that piecewise discontinuous functions are sought. Thus, although $\phi_{i}^{k}=1 / n_{\mathrm{en}}(p)$ for all $k=$ $1, \ldots, n_{\mathrm{en}}(p)$ complies with Equation (5), it is not valid. An obvious choice is to impose $\phi_{i}^{k}=\delta_{i k}$, but it is independent of the subcell size. Here, the following definition is imposed:

$$
\phi_{i}^{k}=\frac{1}{\operatorname{meas}\left(\Omega_{e}^{k}\right)} \int_{\Omega_{e}^{k}} N_{i}(x) \mathrm{d} \Omega \quad \text { for every } i \text { and } k=1, \ldots, n_{\mathrm{en}}(p) .
$$

Note that with this definition the contribution to node $\boldsymbol{x}_{i}$ of the piecewise constant shape function $\phi_{i}(\boldsymbol{x})$ from subcell $\Omega_{e}^{k}$ is equivalent to the contribution of the standard shape function $N_{i}(\boldsymbol{x})$.

\subsection{Selection of parameter $\alpha$}

A major advantage of DG methods is their flexibility in modifying the approximation space element by element. In steady-state problems such as the ones studied here, the value of $\alpha$ is adapted to adequately capture the different discontinuities in the solution. Moreover, in transient problems, also studied here, the parameter $\alpha$ adapts its value in space and in time to accurately capture strong variations of the solution. Thus, without modifying the mesh topology, nor the number of DOFs, nor the node position, nor the structure of the matrices, in a very simple manner (a linear variation)

the space of approximations is adapted in space and time to the regularity of the solution.

It is clear from Equation (3) that extreme values of $\alpha$ ( $\alpha=0$ or 1 ) give the well-known cases of a finite volume approximation and a standard continuous DG approximation, respectively. For intermediate values of $\alpha$, the shape functions introduce jumps of different magnitude across the
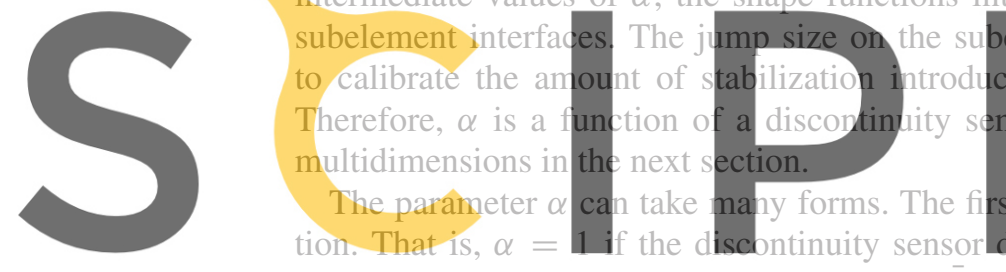

tion. That is, $\alpha=1$ if the discontinuity sensor
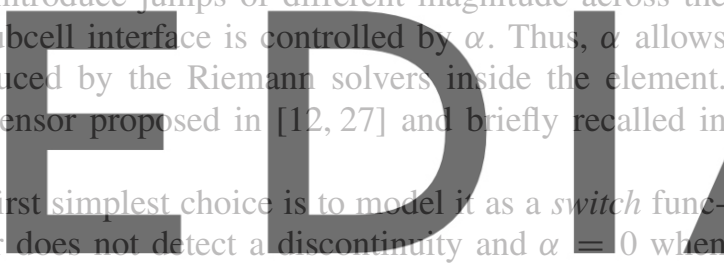

a discontinuity is detected. Thus, functions $\bar{N}_{i}$ are prescribed to be either piecewise constant or

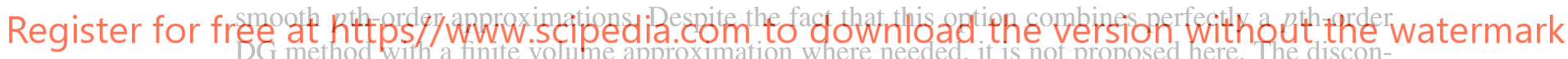

tinuity sensor used here is very accurate and efficient but it does not have the precision to switch drastically from DG to finite volumes. Moreover, as discussed in [28,29] the use of discontinuous functions can severely hamper the convergence of Euler and Navier-Stokes codes to steady state.

In fact, a linear variation is proposed. This improves convergence to steady state and also introduces a smooth transition between DG and finite volumes. In this manner when the discontinuity sensor clearly detects a discontinuity $\alpha=0$ and when the solution is clearly smooth $\alpha=1$. In between a linear variation progressively introduces larger discontinuities. Note however, that a $p$ th-order approximation is only recovered for values of $\alpha=1$. Figure 3 shows both choices.

\subsection{Discontinuity sensor}

The discontinuity sensor defined element by element to determine the parameter $\alpha$, is proposed in $[12,27]$. It only depends on a physical quantity, the Mach number appears to be a reliable sensing variable compared with other physical quantities like the entropy or density, as suggested in [12,30]. Obviously, other choices are possible. It is an element-based projection leading to a single, scalar measure of the smoothness of the numerical approximation. This indicator is a nonlinear operator $S^{e}(s): \Omega_{e} \longrightarrow \mathbb{R}$, depending on the sensing variable $s$, which here is the Mach number, that is, $s=M=\|v\| / c$.

The solution is written within each element in terms of a hierarchical family of orthonormal polynomials determine a suitable sensor for discontinuities. In one dimension (1D), orthonormal Legendre polynomials are used, whereas in two dimensions (2D), an orthonormal Koornwinder basis [31] is employed. For simplicity the sensor is first presented in 1D before it is generalized to 


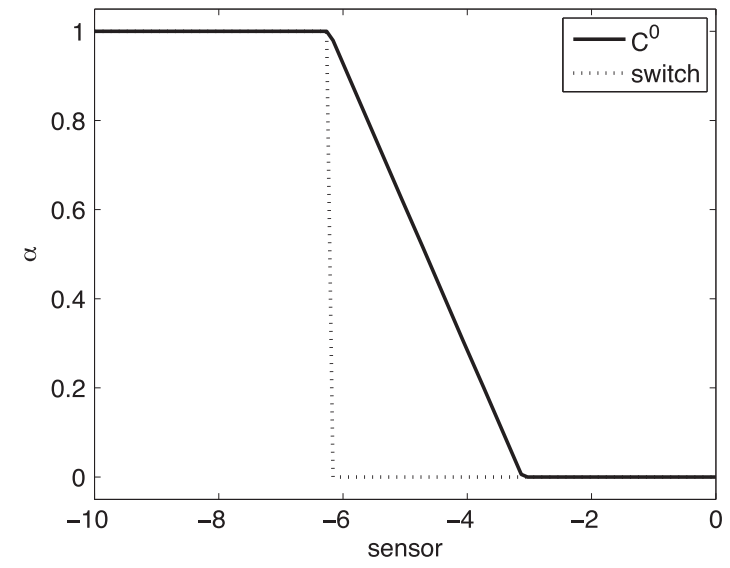

Figure 3. Variation of parameter $\alpha$ with the discontinuity sensor.

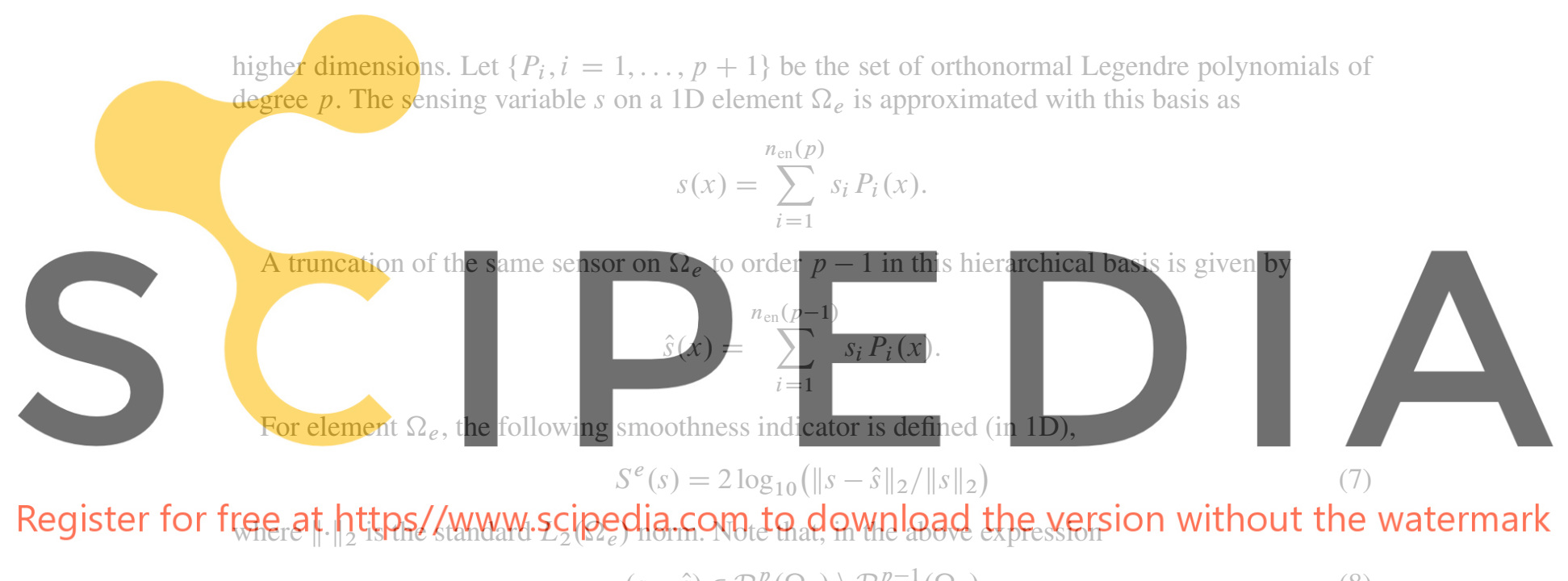

$$
(s-\hat{s}) \in \mathcal{P}^{p}\left(\Omega_{e}\right) \backslash \mathcal{P}^{p-1}\left(\Omega_{e}\right)
$$

and it is a measure of the highest frequencies in the polynomial approximation; in fact, it only contains the higher-order terms of the expansion of $s(x)$.

In what follows, the orthonormality of the basis is exploited to simplify Equation (7) and to further obtain an expression generalizable to multiple dimensions. Note that because of the orthonormality of the Legendre polynomials, the $L_{2}\left(\Omega_{e}\right)$ norm of $s(x)$ becomes $\|s\|_{2}=\sqrt{\mathbf{s}^{T} \mathbf{s}}$, where vector $\mathbf{s}$ is defined by the coefficients of the polynomial expansion, that is, $\mathbf{s}^{T}=\left(s_{1}, \ldots, s_{n_{\mathrm{en}}(p)}\right)$. Thus, given the hierarchy of the polynomial basis, Equation (7) can be rewritten as

$$
S^{e}(s)=\log _{10}\left(s_{n_{\mathrm{en}}(p)}^{2} / \mathbf{s}^{T} \mathbf{s}\right)
$$

The discontinuity sensor in higher dimensions works analogously. The orthonormal Legendre basis is replaced by the orthonormal Koornwinder one. As noted previously (see (8)), $(s-\hat{s})$ is the projection of the high-order approximation $s(\boldsymbol{x})$ on the space of monomials of degree equal to $p$. Thus, Equation (7), or equivalently the multidimensional generalization of (9), becomes

$$
S^{e}(s)=\log _{10}\left(\mathbf{s}^{T} \mathbf{P}_{\mathrm{H}} \mathbf{S} / \mathbf{s}^{T} \mathbf{s}\right)
$$

where $\mathbf{P}_{\mathrm{H}}$ is an orthogonal projection matrix onto the space of monomials of degree $p$. If the orthonormal Koornwinder basis is properly ordered

$$
\mathbf{P}_{\mathrm{H}}=\operatorname{diag}(\overbrace{0 \cdots 0}^{n_{\mathrm{L}}} \overbrace{1 \cdots 1}^{n_{\mathrm{H}}}),
$$


where $n_{\mathrm{L}}$ is the number of lower modes, that is the number of DOFs for polynomials of degree $p-1$ (ex.: $n_{\mathrm{L}}=p(p+1) / 2$ in $\left.2 \mathrm{D}\right)$, and $n_{\mathrm{H}}$ is the number of remaining high-order modes, that is, the number of monomials of degree $p$ (ex.: $n_{\mathrm{H}}=p+1$ in 2D). See Remark 2 if, instead of an orthonormal basis, a more usual nodal basis is used.

This indicator, either Equation (9) for the 1D case or Equation (10) in multidimensions, treats the higher-order solution as though it were comprised of a sequence of Fourier modes. For smooth flows, the coefficients of increasing Fourier modes are expected to decay very quickly, according to $1 / p^{2}$. However, a slower rate of decay indicates the presence of a nonsmooth solution feature. This idea is strongly related to the error indicators used in spectral $h p$-methods (see [32]). Moreover, The strength of the discontinuity dictates the rate of decay of the expansion coefficients (see, e.g. [33]). Figure 4 shows the decay rate of the expansion coefficients for functions a step function and a smooth one, namely: $f(x)=\sin (x+y)$ for $(x, y) \in[-\pi, \pi] \times[-\pi, \pi]$.

In fact, the expected decay defines the threshold for nonsmooth solutions. If the decay of consecutive orders is larger than $1 / p^{2}$, that is, if $S^{e}(s)>S_{0}=-4 \log _{10}(p)$, the solution is assumed nonsmooth and $\alpha=0$. When the decay is clearly below the theoretical one, that is, if $S^{e}(s)<S_{1}=-4 C \log _{10}(p)$, the solution is assumed smooth and $\alpha=1$. When $C=1$ a switch $\alpha$ function is used (see Figure 3). If a linear variation between $\alpha=1$ and $\alpha=0$ is sought $C$ is chosen larger than one. Because results are very robust for small variations of $C$, in practice, that is, for all the examples shown, $C=2$ is imposed with good results. These two thresholds are also depicted in Figure 4 .

Finally, it must be noted that this discontinuity sensor, originally proposed in [12], has been found to be extremely reliable for the Euler equations and medium to high orders of approximation (i.e. $R \geqslant 2$ )
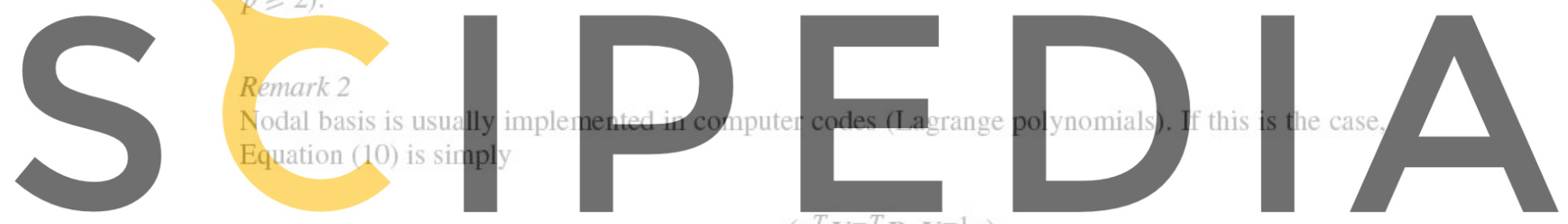

Register for free at https//www.scipedia.com to downlload the version without the watermark

where $\mathrm{V}$ is the Vandermonde matrix whose inverse maps the Lagrange basis onto the orthonormal one (see Section 3.1 in [34] and references therein for a detailed discussion on these transformations).

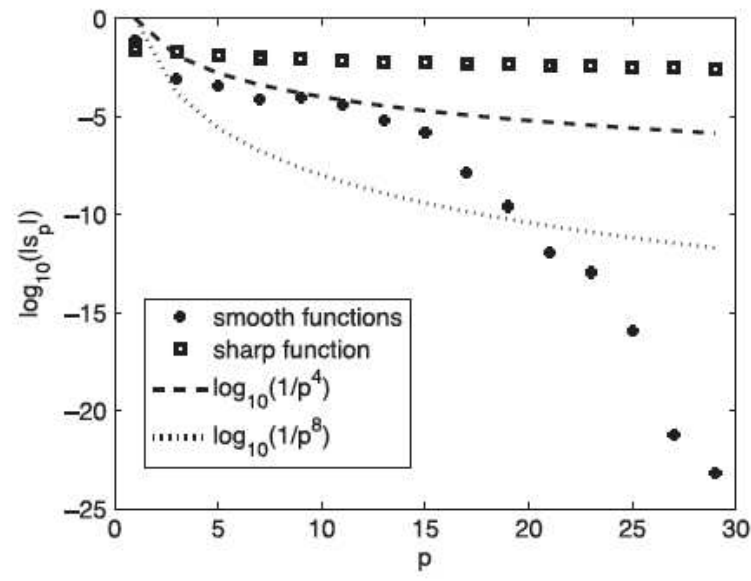

Figure 4. Decay rate of the expansion coefficients for a step function and a smooth 2D function and thresholds $S_{1}=\log _{10}\left(1 / p^{8}\right)$ and $S_{0}=\log _{10}\left(1 / p^{4}\right)$. 


\section{NUMERICAL TESTS}

This section presents several numerical examples of compressible flow for both transonic and supersonic flow regimes, and transient and steady-state problems. Coarse meshes and high-order approximations are used to assess the performance of the proposed methodology. Moreover, the locality of this approach is also shown. All the tests are performed with triangular meshes and with an explicit Runge-Kutta time integration scheme. For steady-state solutions, the relative $L_{2}$ norm of the density residual is taken as a criterion to test convergence.

\subsection{The shock tube problem}

The first test to validate this methodology is the classical shock tube problem proposed in [35] for which there exists an exact solution to the 1D Euler equations. The problem involves a shock wave, a contact discontinuity and an expansion fan. The problem is solved in a 2D setting with a computational domain of dimensions $\Omega=10,1[\times 10,0.4[$. The following initial data are used:

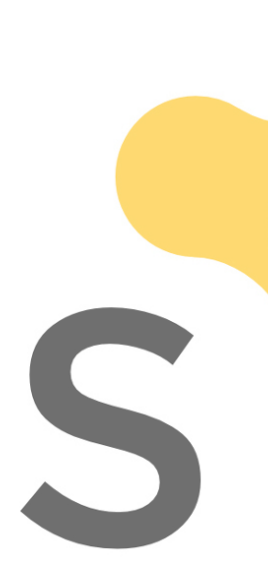

$$
\begin{aligned}
0 & <x_{1} \leqslant 1 / 2 \\
\rho & =1.0 \\
\rho v & =0.0 \\
\rho E & =2.5
\end{aligned}
$$$$
1 / 2<x_{1} \leqslant 1
$$$$
\rho=0.125
$$$$
\rho v=0.0
$$$$
\rho E=0.25
$$

The initial density and pressure difference are maintained by a diaphragm, which is ruptured at $t=0$. A uniform m As notect earkier, this is computations using The purpose of th lem and to show the is computed trpto $t$ clearly develoned. Note that in atransient problenin.
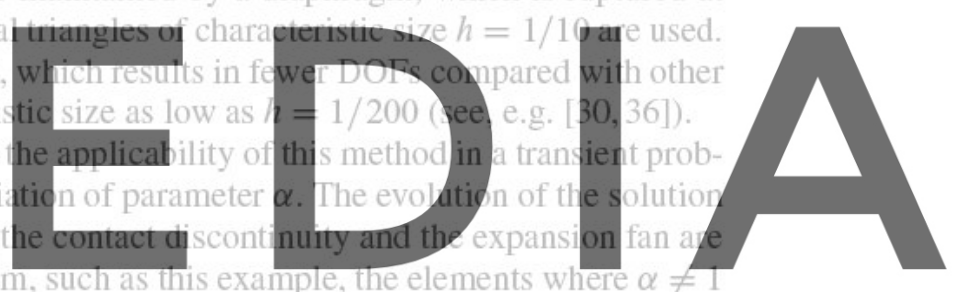

evolve with time. Capturing the correct wave speeds shows a good performance of this technique.

Register for free atehttips/ $/$ www s scipedia.comctodownload the version withoutnthe- watermark ity sensor. Figure 5 shows the three different choices, namely, a linear variation between $S_{0}$ and $S_{1}$,

$$
\alpha_{0}(S)= \begin{cases}1 & \text { if } S<S_{1}, \\ \left(S_{0}-S\right) /\left(S_{0}-S_{1}\right) & \text { if } S_{1} \leqslant S<S_{0} \\ 0 & \text { if } S_{0} \leqslant S,\end{cases}
$$

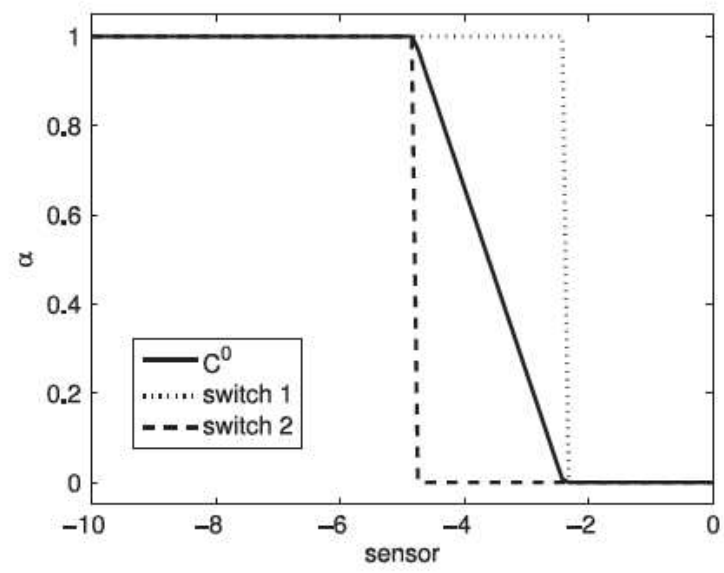

Figure 5. Shock tube problem, $\alpha$ functions tested. 
and two switch functions,

$$
\alpha_{1}(S)=\left\{\begin{array}{ll}
1 & \text { if } S<S_{1}, \\
0 & \text { if } S_{1} \leqslant S,
\end{array} \text { and } \alpha_{2}(S)= \begin{cases}1 & \text { if } S<S_{0} \\
0 & \text { if } S_{0} \leqslant S\end{cases}\right.
$$

To analyze the results density and pressure are shown along a section following the flow direction (i.e. projecting the solution on the plane $x_{2}$ and the corresponding variable). Figures 6-8 show the results for the different choices of the $\alpha$-function. Velocity and energy results produce similar conclusions (for a detailed error analysis between the different switches, see [37]).

These figures show that the position of the flow discontinuities is well predicted in the three cases. Nevertheless, the $\alpha_{1}$ switch (see Equation (12)) clearly introduces excessive numerical diffusion, see Figure 7. Whereas oscillations in Figure 8 clearly indicate that the $\alpha_{2}$ switch, on the contrary, is under-diffusive. These oscillations are more obvious in the $x_{1}$ component of the velocity (see Figure 9). In summary, imposing piecewise constant approximations when the decay in the last polynomial order is not below $1 / p^{4}$ introduces excessive numerical diffusion, and oscillations are present when a $p$-th order continuous approximation is assumed if the decay is just $1 / p^{2}$ or larger. The linear variation determined by $\alpha_{0}$ (see Equation (11)) induces accurate results as shown in Figure 6. It is important to recall that these solutions are obtained with a coarse $(h=1 / 10)$ high-order $(p=6)$ mesh.

\subsection{Supersonic flow past a bump}

The supersonic flow past a bump is a well-known benchmark test for steady-state simulation at different Mach numbers (see, e.g. [38-40]). It consists of inviscid flow in a channel with a 4\%
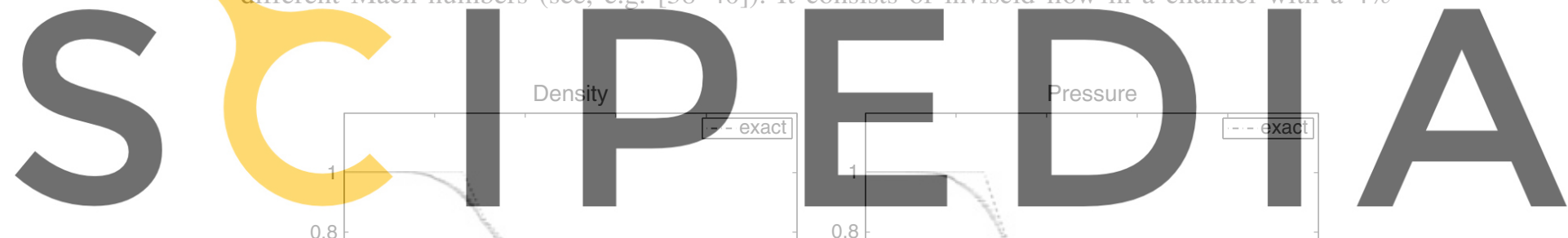

Register for free at. $\mathrm{https} / / \mathrm{www}$.scipedia.com to dogwnload the version without the watermark
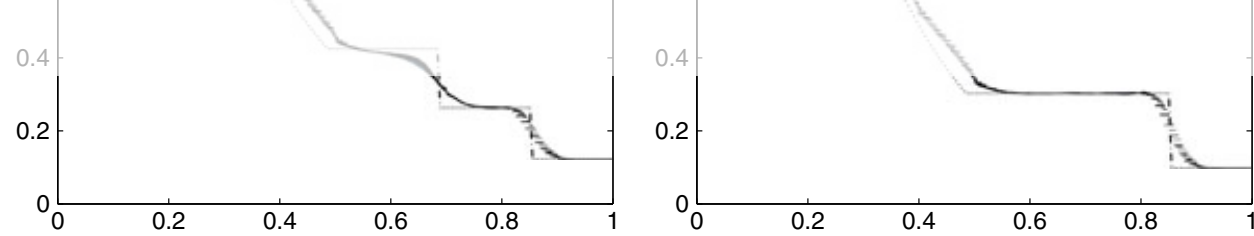

Figure 6. Shock tube profiles for $\alpha_{0}$.
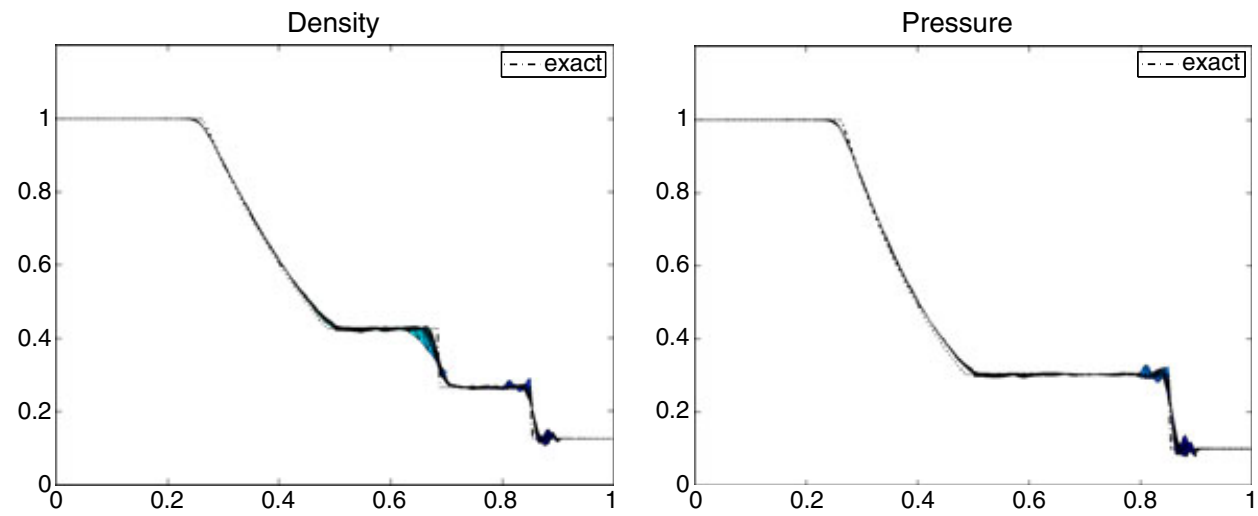

Figure 7. Shock tube profiles for the first switch: $\alpha_{1}$. 

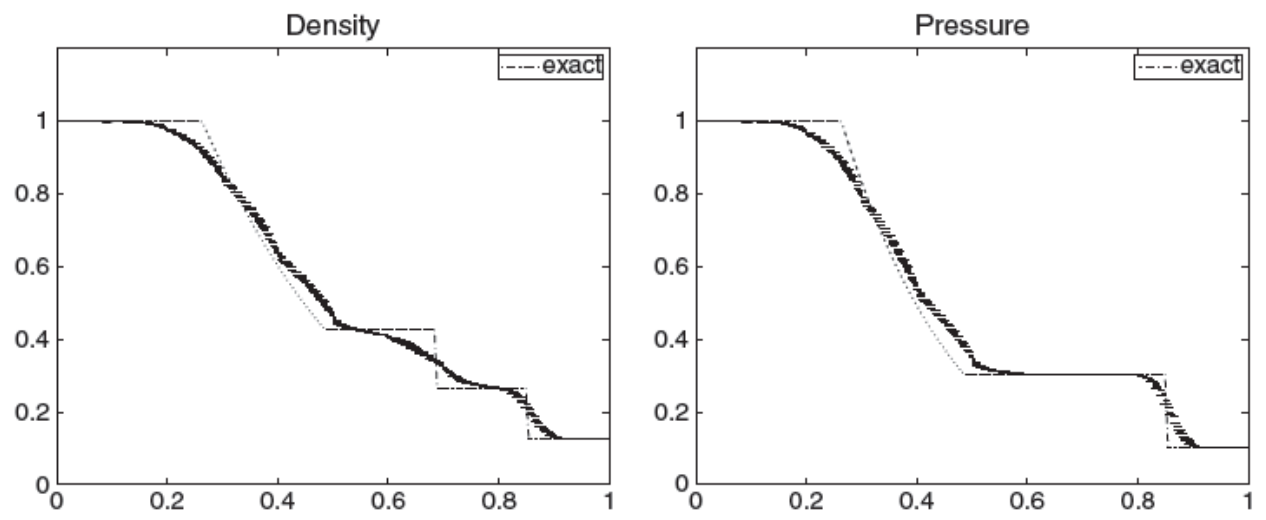

Figure 8. Shock tube profiles for the second switch: $\alpha_{2}$.

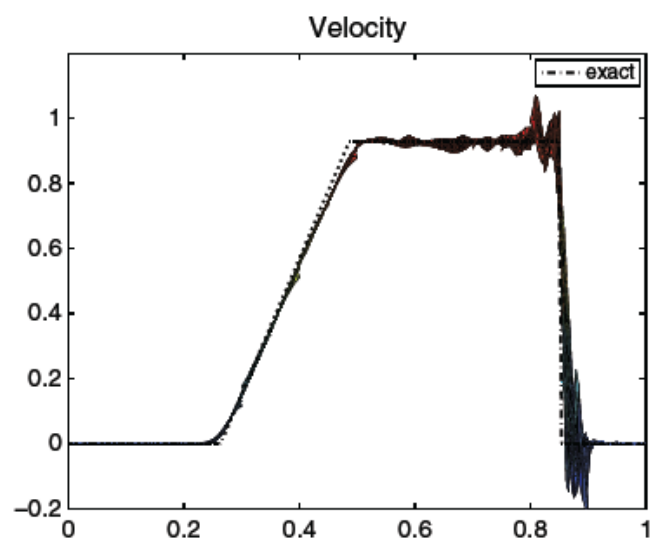

Figure 9. Shock tube $v_{1}$ profile for the second switch: $\alpha_{2}$.

thick circular bump on the bottom. The channel is 3 units in length and 1 unit in height. The bump is located in the middle of the channel with chord length equal to 1 . Inflow boundary condition is applied on the left, and outflow boundary condition on the right. At the top and bottom of the domain, solid wall boundary conditions are considered. The inlet Mach number is $M=1.4$. In this case the steady-state solution is characterized by two stagnation points and a change in the direction of the shock because of the reflexion on the right boundary.

Common approaches use FV schemes combined with an adaptive unstructured mesh refinement (see, e.g. [38]). Here, high-order approximations with relative coarse, uniform and structured meshes are used to show the applicability of the proposed methodology. The first approximation is computed over a fine computational mesh of 1452 elements and degree $p=3$ (total number of DOFs 58080 ) and the second one is computed over a coarse mesh of 588 elements with degree $p=5$ (total number of DOFs 49392). Both meshes are depicted in Figure 10.

Figures 11 and 12 depict the Mach number obtained with both discretizations. Qualitatively, the solutions are similar, showing stable approximations with sharp shock profiles free of spurious

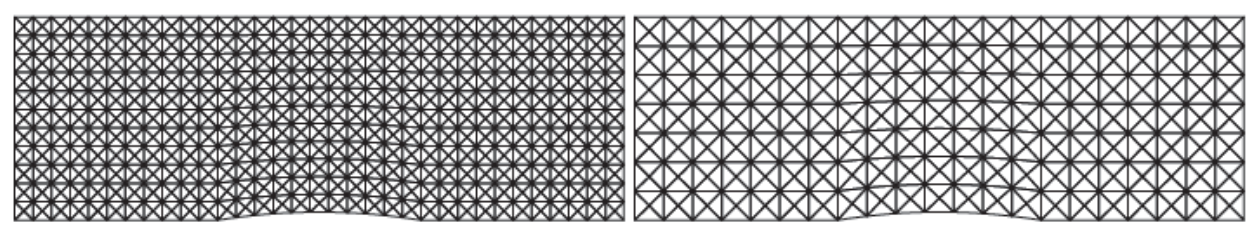

Figure 10. Supersonic flow past a bump with $M=1.4$ : computational meshes. On the right, a fine mesh of 1452 elements and on the left a coarse one of 588 elements. 

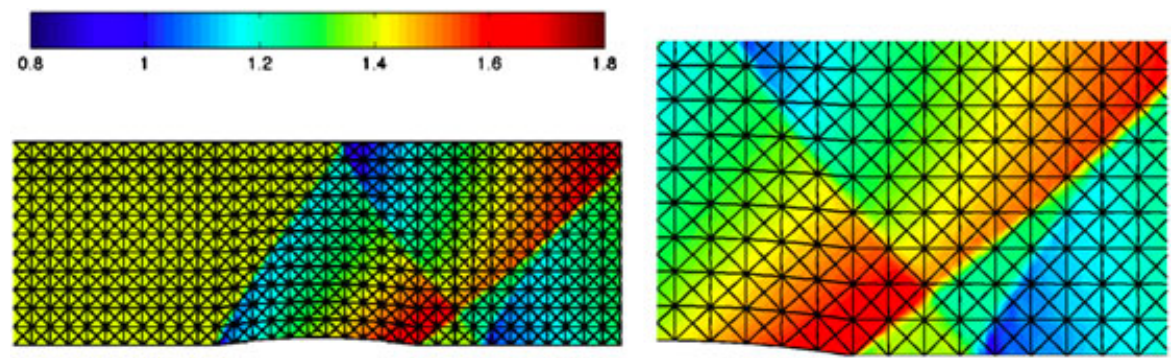

Figure 11. Supersonic flow past a bump with $M=1.4$ : Mach number for $p=3$ and 1452 elements.
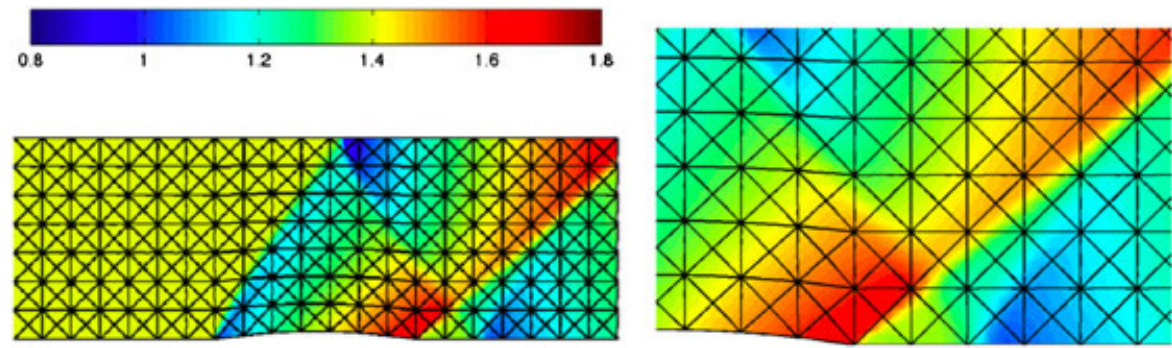

Figure 12. Supersonic flow past a bump with $M=1.4$ : Mach number for $p=5$ and 588 elements.

oscillations. A detail of the oblique shock behind the duct is also shown to illustrate that shocks are captured essentially inside one element, with independence of the mesh geometry. This is specially relevant for the fifth-order approximation, where the element size doubles the size of the third-order mesh and the shock is not aligned with edges. Moreover, in this case, it is clear that the shock width is far more thinner than the element size.

A horizontal section along $y=0.4$ is shown in Figure 13 to compare the different solutions in more detail. The solutions for $p=3$ and $p=5$ are also compared with a linear approximation on a very fine mesh. The linear mesh requires 14000 elements, that is, a triple number of DOFs, to obtain similar accuracy of the $p=5$ mesh. The comparison reveals that a sharper profile is obtained with the high-order solution of degree $p=5$ and a coarse mesh than with the low-order one of $p=3$ and fine mesh. Hence, using large elements and increasing the degree of approximation (not the number of DOFs) accuracy is improved. Compared with other high-order techniques that also use coarse meshes (see, e.g. [14]), the present approximation is able to obtain accurate results with uniform coarse meshes and fewer DOFs, avoiding adaptivity of the mesh along the shock regions.

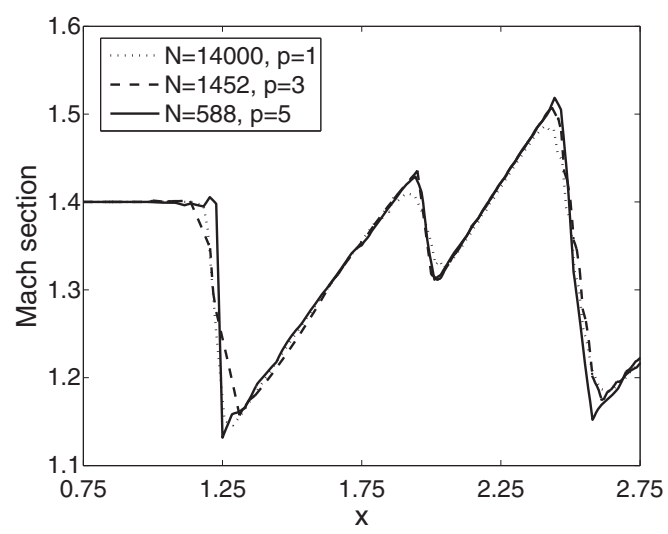

Figure 13. Supersonic flow past a bump with $M=1.4$ : distribution of the Mach number along section $y=0.4$. 
The distribution of parameter $\alpha$ at steady-state is shown in Figure 14. Elements in blue correspond to $\alpha \neq 1$ and consequently elements where accuracy is reduced to order $h / p$. Note that these areas coincide, as expected, with sharp gradients in Mach number; that is, along the shock front. Notice that in contrast with standard approaches, the mesh is not modified neither refined along the shock region. Hence, the computational cost of recomputing the mesh within each iteration is avoided.

\subsection{Transonic flow in a converging-diverging nozzle}

The converging-diverging nozzle is another standard benchmark test involving the flow of inviscid nonheat-conducting air through a nozzle (see, e.g. [41,42]). Here, the 2D (planar) case is considered [43]. The computational domain $\Omega$ is in $] 0,3[\times] 0,1[$ and presents a variable section defined by a sinusoidal profile. Inviscid wall boundary conditions are imposed on the lower and upper edges of the domain. Inflow conditions impose the Mach number, $M=0.5$, density and pressure, both equal to 1 . At the supersonic outflow a pressure condition, the ratio between the stagnation and the free-stream pressure, is prescribed equal to 0.67 . Under these circumstances supersonic flow with a normal shock in the diffusing section is created.

Two different discretizations are considered: 916 elements and degree $p=3$ (36640 DOFs) and 278 elements with degree $p=5$ (23352 DOFs). Note that both discretizations use uniform meshes and a reduced number of DOFs, for instance an order of magnitude lower than in [43].

Figure 15 depicts the Mach number for the third-order approximation. In spite of the coarse approximation and the uniform mesh, steady state is reached and the shock is captured avoiding spurious oscillations. However, a closer look reveals some lack of accuracy: the shock loses its typical bow shape and it is influenced by the discretization.

Obviously, mesh adaption near the shock would alleviate these inaccuracies. However, high-order approaches may also be an alternative thanks to the discontinuous basis proposed here. In fact, the solution with $p=5$, shown in Figure 16, presents accurate results and a clearly defined bow shock independent of the discretization. The shock is clearly captured inside the elements and with thickness less than the element size. Note also, that the higher order approach requires a reduced number of DOF.

In Figures 17 and 18 the Mach number distributions along the horizontal (at $y=0.5$ ) and vertical (at $x=2$ ) section are depicted. Moreover, the high-order solutions are further compared with a FV computation. A uniform mesh is also employed for the FV case to do a fair comparison. More than
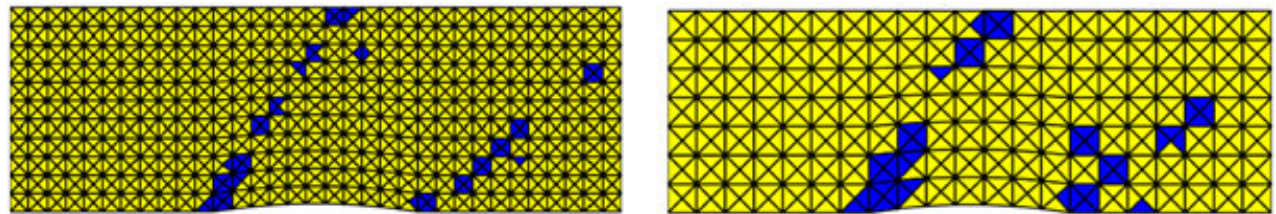

Figure 14. Supersonic flow past a channel with $M=1.4$ : distribution of $\alpha$ for $p=3$ and 1452 elements (left) and $p=5$ and 588 elements, yellow for $\alpha=1$, blue for $0<\alpha<1$, and red for $\alpha=0$.
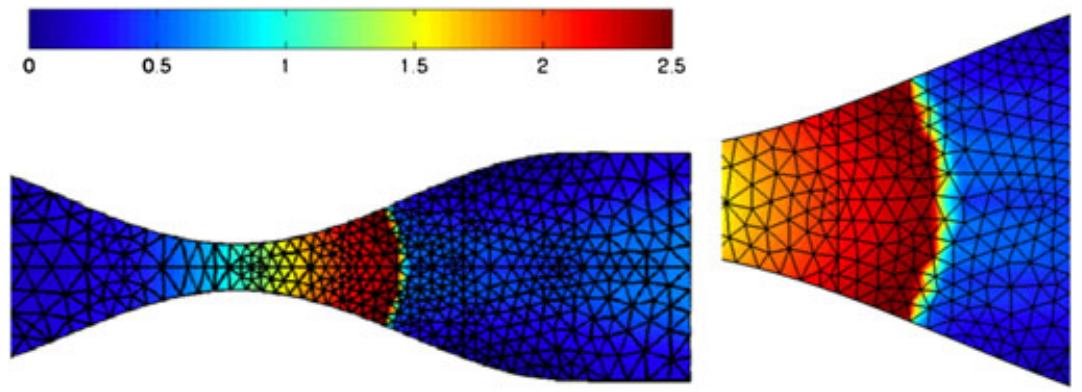

Figure 15. Transonic nozzle: Mach number for a solution computed with $p=3$ and a mesh of 916 elements. Detail of the shock on the right. 

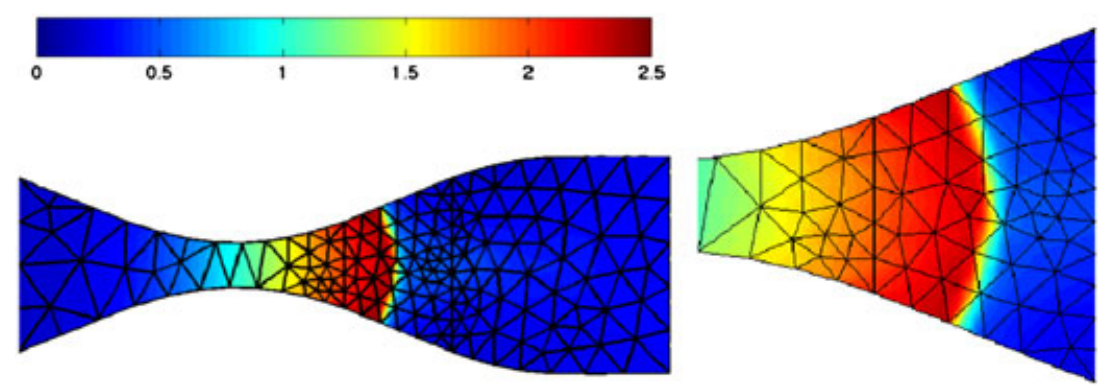

Figure 16. Transonic nozzle: Mach number for a solution computed with $p=5$ and a mesh of 278 elements. Detail of the shock on the right.

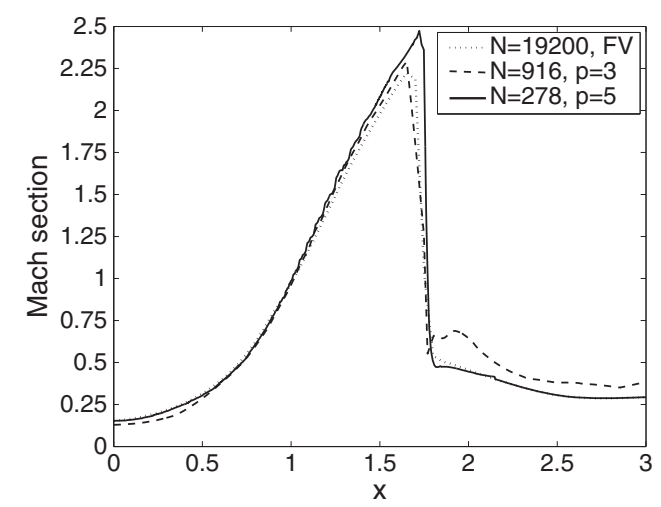

Figure 17. Transonic nozzle: Mach number profiles along $y=0.5$ for $p=0,3$ and 5.

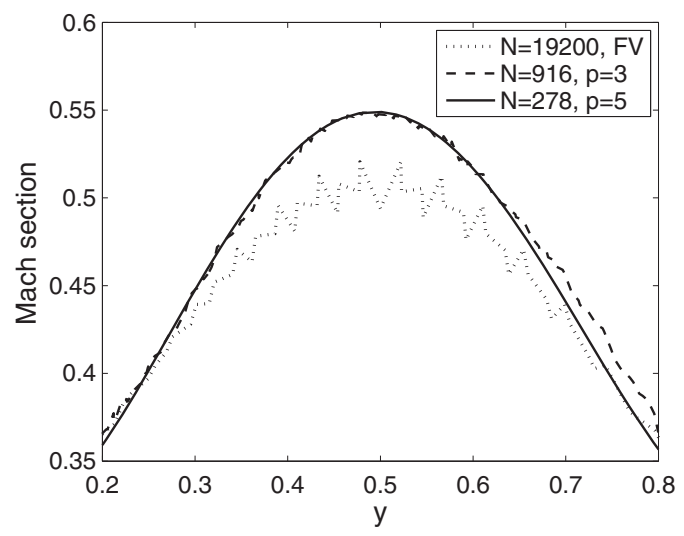

Figure 18. Transonic nozzle: Mach number profiles along $x=2$ for $p=0,3$ and 5 .

twice the number of DOFs has been used (19200 elements, that is, 76800 number of DOFs) and as shown they are not sufficient. Figure 17 clearly shows that the $p=5$ solution, which is the one with lower DOF, presents the sharpest shock. The FV and $p=3$ solutions are rather coincidental, but the $p=3$ solution presents a small oscillation behind the shock. However, Figure 18 reveals interesting conclusions. The FV approximation results in nonphysical variations of the Mach number, although the transversal shock profile was smooth, the $p=3$ solution has lost its symmetry, and the $p=5$ results are the expected ones. 


\subsection{The forward-facing step problem}

A challenging test for computing unsteady shock waves is the numerical simulation of a wind tunnel with a flat faced step, originally introduced in [44] to compare several difference schemes in classical fluid dynamics. It is now a standard numerical benchmark (see, e.g. [5, 20,45-47]). The problem under consideration is a Mach 3 flow in a wind tunnel of 3 units in length and 1 unit in width. The step is 0.2 units high and it is located at 0.6 units from the left end of the tunnel. Initially the tunnel is filled with a gas, with adiabatic coefficient $\gamma=1.4$, which everywhere has density $\rho=1.4$, pressure $p=1$, and velocity $v=(3,0)^{T}$. Solutions are shown at time $t=4$ of the computations, where a special and complicated shock configuration is shown.

An inflow boundary condition is applied at the left end of the computational domain and outflow boundary condition at the right end. Along the walls of the tunnel, and on the boundary marked by the step, inviscid wall boundary conditions are applied. The corner of the step is the center of a rarefaction fan and, thus, a singular point of the flow. It is well known that this singularity leads to an erroneous entropy layer at the downstream bottom wall, and spurious Mach oscillations at the top wall next to the Mach stem. Unlike in [45] and many other references, here the scheme is not modified near the corner, neither the mesh is refined. This will allow us to see the influence of large elements with high-order approximation in the entropy layer. Note that results are very reasonable although no modification near this corner is introduced.

Two meshes with different approximations are compared: a very fine mesh of 10677 elements and $p=2$, and a coarse mesh of 1728 elements and degree $p=6$. Both meshes are depicted in Figure 19. Notice that both are uniform with no special refinement around the corner or at the shocks.

Figure 20 shows the computed density profiles at time $t=4$ for both meshes. The location and shape of the shocks are consistent in both computations. The shocks and the rarefaction fan, which is created around the corner, are well-captured without generating relevant noise. This is specially remarkable around the upper slip line from the triple point and behind the Mach stem, compared to other results $[5,20]$. The contact discontinuity arising from the triple point is slightly better resolved with the high-order approximation despite fewer DOFs are involved.

Figure 21 shows the map of $\alpha$, which controls the discontinuities inside each element, for each element and both approximations. Again, the results are consistent: the detection is aligned with the major shocks and clearly identifies the sharp $\lambda$-type shock. Because the elements in the $p=6$ mesh are coarser than the $p=2$ mesh, the thickness of the detected region is bigger for the high-order approximation. This fact is a consequence of the element-by-element behavior of the sensor and the parameter $\alpha$. Nevertheless, as shown next, this does not imply that the shock width is larger for the coarser mesh $(p=6)$.
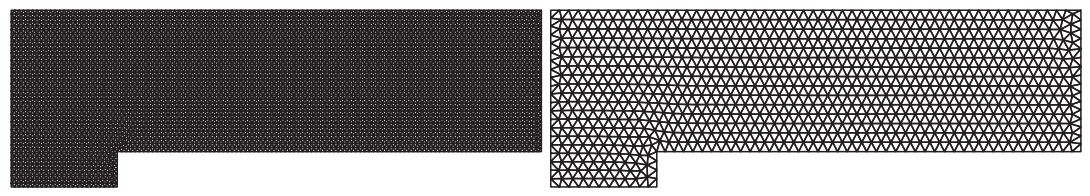

Figure 19. Forward-facing step with Mach 3: computational meshes of 10677 elements (left) and 1728 elements (right).

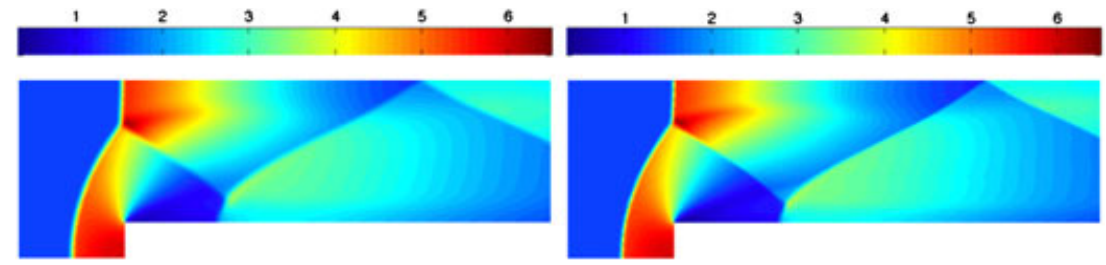

Figure 20. Forward-facing step with Mach 3: density profile for $p=2$ and 10677 elements (left) and $p=6$ and 1728 elements (right). 


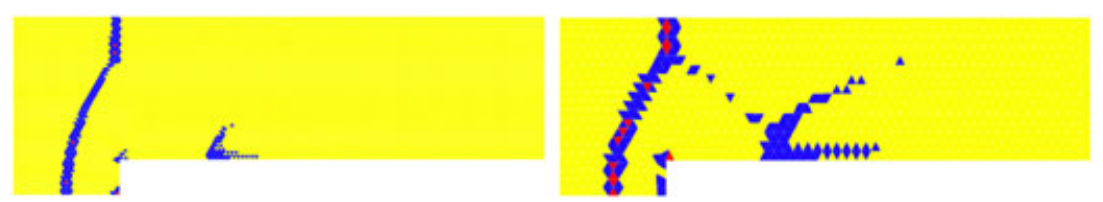

Figure 21. Forward-facing step with Mach 3: $\alpha$ values for $p=2$ and 10677 elements (left) and $p=6$ and 1728 elements (right), yellow for $\alpha=1$, blue for $0<\alpha<1$, and red for $\alpha=0$.

Density along $y=0.2$ is shown in Figure 22 to better compare both solutions and their accuracy. Both cases locate the bow shock at $x=0.3$ approximately. However, a sharper front is observed for $p=6$; that is, although the $p=6$ has a coarser mesh size, the shock profile is thinner. Moreover, the $p=6$ solution does not present oscillations, while the $p=2$ shows small oscillations near the outflow boundary and bigger ones just after the shock, at $x=0.6$, where the density drops by a factor of 5 .

The entropy production in the vicinity of the step corner, caused by the singular point is a clear indicator of the noise introduced by the scheme and also its ability to deal with singularities. Adaptive refinement near the singular point is advocated in $[5,46]$ to obtain more accurate solutions (with less entropy production). Other alternatives are possible using a specific numerical treatment on the singular point (see, e.g. [45]). Here, no adaptivity or entropy manipulation is done to see the influence of high-order approaches. The adiabatic constant, $A=p / \rho^{\gamma}$, which is a function of entropy, is plotted in Figure 23. Note that the amount of entropy generation along the downstream boundary is not excessive and moreover that it is drastically reduced for $p=6$.

To get a better insight on the performance of the proposed method, a sequence of four uniform meshes, shown in Figure 24, is used with the same order of approximation, $p=4$. Figure 25 shows the density along $y=0.2$. Obviously, accuracy improves as the mesh is refined and a sharper shock is obtained with finer meshes. Nevertheless, even for the coarse mesh results are reasonable. However, it is important to note that results converge as the mesh is refined.

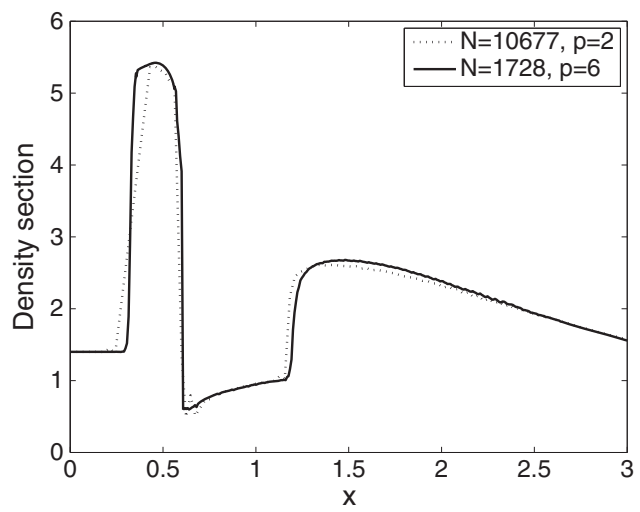

Figure 22. Forward-facing step with Mach 3: section along $y=0.2$ of the density profile.
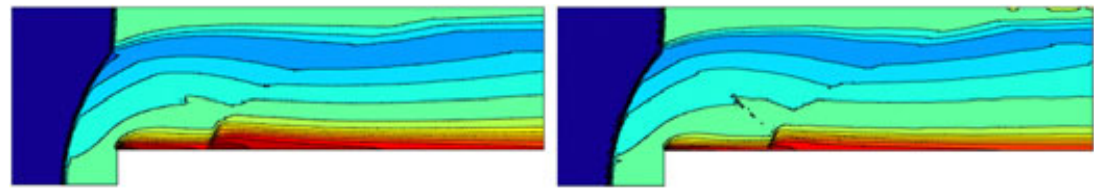

Figure 23. Forward-facing step with Mach 3: contours of adiabatic constant $A=p / \rho^{\gamma}$ for $p=2$ and 10677 elements (left) and $p=6$ and 1728 elements (right). 

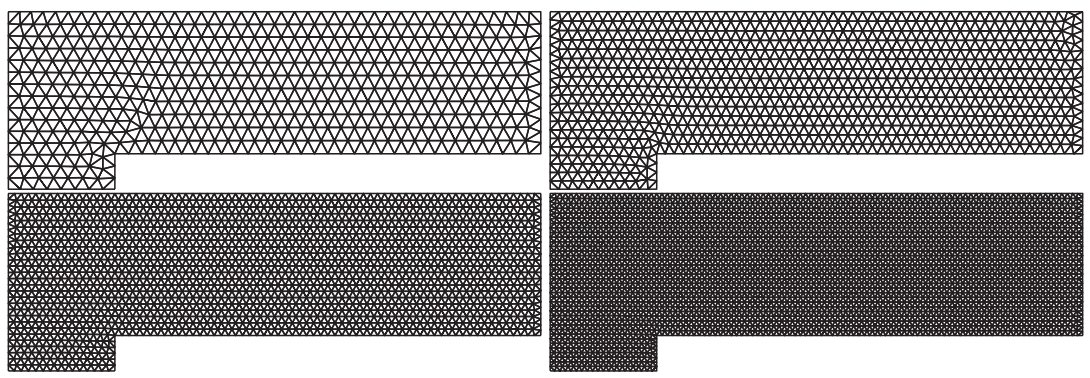

Figure 24. Forward-facing step with Mach 3: computational meshes. From left to right and top to bottom: $915,1728,3457$ and 6286 elements.
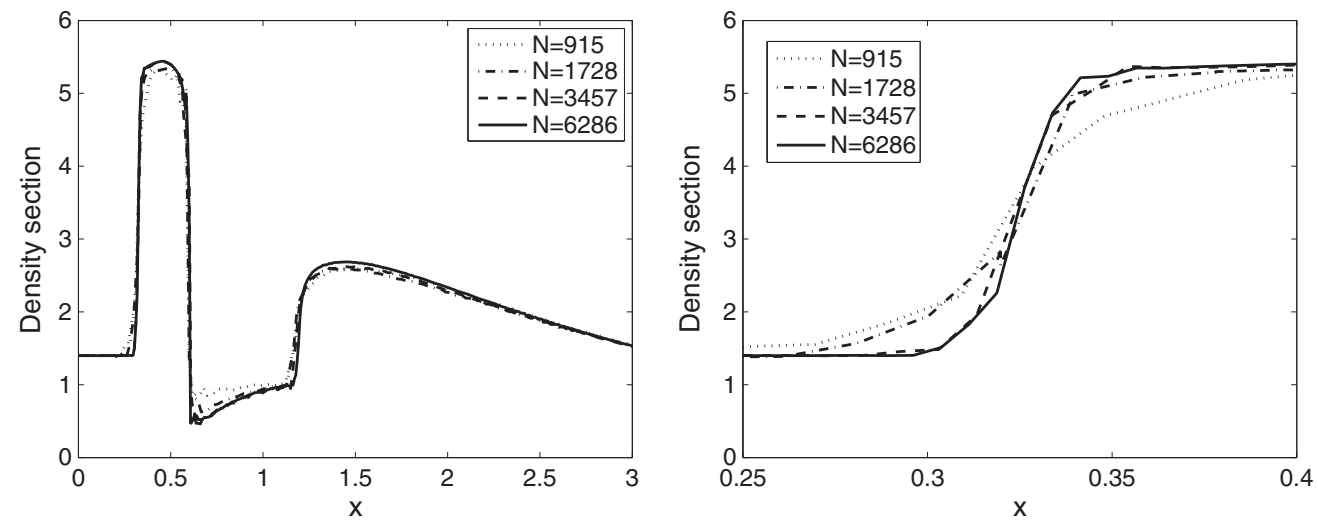

Figure 25. Forward-facing step with Mach 3: section along $y=0.2$ of the density profile and detail of the first shock.

\section{CONCLUSIONS}

Discontinuous Galerkin (DG) methods usually settle for mesh adaptivity to capture shocks and discontinuities. In the framework of high-order approximations for high-fidelity computations, this recourse implies an important computational overhead. Recall that DG methods duplicate nodes. Here, a new shock-capturing technique is proposed. This novel approach does not require mesh adaption, does not increase the number of DOFs, and does not change the topology of the mesh. It exploits inherent stabilization properties of numerical fluxes in DG introducing discontinuities inside the required elements. It is based on one parameter that automatically modifies the shape functions from continuous high-order ones to discontinuous ones depending on the smoothness of the solution. Obviously, the high-order accuracy is reduced to order one in the shock vicinity, but the shock width is of order $h / p$ (where $h$ is the characteristic element size and $p$ the order of the approximation).

Results show the robustness of this method, which does not require any tuning and provides a stable solution with sharp resolution of shocks within the elements. The unsteady example shows that this method allows to capture wave speeds with the required precision. Moreover, the steady examples confirm coarse meshes that narrow, and well-resolved shocks are computed independently of the complexity of the shock structure. Finally, it is important to note that numerical results show that high-order elements require fewer DOFs compared with low-order approximations when high accuracy is necessary.

\section{ACKNOWLEDGEMENTS}

This work was supported by the Ministerio de Ciencia y Tecnología; BIA2007-66965 and DPI2007-62395. 


\section{REFERENCES}

1. Bassi F, Rebay S. A high-order accurate discontinuous finite element method for the numerical solution of the compressible Navier-Stokes equations. Journal of Computational Physics 1997; 131(2):267-279. DOI: 10.1006/jcph.1996.5572.

2. Cockburn B, Shu C-W. TVB Runge-Kutta local projection Discontinuous Galerkin finite element method for conservation laws. II. General framework. Mathematics of Computation 1989; 52(186):411-435. DOI: 10.2307/2008474.

3. Biswas R, Devine KD, Flaherty JE. Parallel, adaptive finite element methods for conservation laws. Applied Numerical Mathematics 1994; 14(1-3):255-283. DOI: 10.1016/0168-9274(94)90029-9.

4. Cockburn B, Shu C-W. The Local Discontinuous Galerkin method for time-dependent convection-diffusion systems. SIAM Journal on Numerical Analysis 1998; 35(6):2440-2463. DOI: 10.1137/S0036142997316712.

5. Cockburn B, Shu C-W. Runge-Kutta Discontinuous Galerkin methods for convection-dominated problems. Journal of Scientific Computing 2001; 16(3):173-261. DOI: 10.1023/A:1012873910884.

6. Krivodonova L, Xin J, Remacle J-F, Chevaugeon N, Flaherty JE. Shock detection and limiting with Discontinuous Galerkin methods for hyperbolic conservation laws. Applied Numerical Mathematics 2004; 48(3-4):323-338. Workshop on Innovative Time Integrators for PDEs.

7. Remacle J-F, Li X, Shephard MS, Flaherty JE. Anisotropic adaptive simulation of transient Flows using Discontinuous Galerkin methods. International Journal for Numerical Methods in Engineering 2005; 62(7):899-923. DOI: 10.1002/nme.1196.

8. Nguyen NC, Peraire J, Cockburn B. An implicit high-order hybridizable Discontinuous Galerkin method for nonlinear convection-diffusion equations. Journal of Computational Physics 2009; 228(23):8841-8855. DOI: 10.1016/j.jcp.2009.08.030.

9. Barth TJ. Aspects of Unstructured Grids and Finite Volume Solvers for the Euler and Navier-Stokes Equations (Part 4), 1995.

10. LeVeque RJ. Finite volume methods for hyperbolic problems. Cambridge Texts in Applied Mathematics, 1 st edn. Cambridge University Press: Cambridge, 2002.

11. Von Neumann J, Richtmyer RD. A method for the numerical calculation of hydrodynamic shocks. Journal of Applied Physics 1950; 21:232-237. DOI: 10.1063/1.1699639.

12. Persson P-O, Peraire J. Sub-cell shock capturing for Discontinuous Galerkin methods. Proc. of the 45th AIAA Aerospace Sciences Meeting and Exhibit: Reno, Nevada, 2006. AIAA-2006-0112.

13. Barter GE, Darmofal DL. Shock capturing with PDE-based artificial viscosity for DGFEM:Part I. Formulation. Journal of Computational Physics 2010; 229(5):1810-1827. DOI: 10.1016/j.jcp.2009.11.010.

14. Premasuthan S, Liang C, Jameson A. Computation of flow with shocks using spectral difference scheme with artificial viscosity. Proc. of the 48th AIAA CAerospace Sciences Meeting including the New horizons Forum and Aerospace Esposition: Orlando, FL, 2010. AIAA-2010-1449.

15. Casoni E, Peraire J, Huerta A. One-Dimensional Shock-Capturing for High-Order Discontinuous Galerkin Methods, ECCOMAS Multidisciplinary Jubilee Symposium, Computational Methods in Applied Sciences, Vol. 14. Springer Netherlands, 2009.

16. LeVeque RJ. Numerical methods for conservation laws, Second edn., Lectures in Mathematics ETH Zürich. Birkhäuser Verlag: Basel, 1992.

17. Cockburn B, Lin S-Y, Shu C-W. TVB Runge-Kutta local projection Discontinuous Galerkin finite element method for conservation laws. III. one-dimensional systems. Journal of Computational Physics 1989; 84(1):90-113. DOI: 10.1016/0021-9991(89)90183-6.

18. Shu C-W, Osher S. Efficient implementation of essentially nonoscillatory shock-capturing schemes. Journal of Computational Physics 1988; 77(2):439-471. DOI: 10.1016/0021-9991(88)90177-5.

19. Qiu J, Shu C-W. Runge-Kutta Discontinuous Galerkin method using WENO limiters. SIAM Journal on Scientific Computing 2005; 26(3):907-929.

20. Zhu J, Qiu J, Shu C-W, Dumbser M. Runge-Kutta Discontinuous Galerkin method using WENO limiters II: Unstructured meshes. Journal of Computational Physics 2008; 227(9):4330-4353. DOI: 10.1016/j.jcp.2007.12.024.

21. Donea J, Huerta A. Finite element methods for flow problems. John Wiley \&amp Sons: Chichester, 2003.

22. Roe PL. Approximate Riemann solvers, parameter vectors, and difference schemes. Journal of Computational Physics 1997; 135(2):250-258. DOI: 10.1006/jcph.1997.5705.

23. Hirsch C. Numerical Computation of Internal and External Flows: Computational methods for inviscid and viscous flows, Wiley series in numerical methods in engineering Volume 2 of Numerical Computation of Internal and External Flows. John Wiley \& Sons: Brussels, Belgium, 1990.

24. Chavent G, Cockburn B. The local projection $P^{0} P^{1}$-Discontinuous-Galerkin finite element method for scalar conservation laws. RAIRO Modélisation Mathématique et Analyse Numérique 1989; 23(4):565-592.

25. Van Leer B. Towards the ultimate conservative difference scheme V. A second order sequel to Godunov's method. Journal of Computational Physics 1997; 135(2):229-248. DOI: 10.1006/jcph.1997.5704.

26. Cueto-Felgueroso L, Colominas I. High-order Finite Volume methods and multiresolution reproducing kernels. Archives of Computational Methods in Engineering 2008; 15(2):185-228. DOI: 10.1007/s11831-008-9017-y.

27. Nguyen NC, Persson P-O, Peraire J. RANS solutions using high order Discontinuous Galerkin methods. Proc. of the 44th AIAA Aerospace Sciences Meeting and Exhibit: Reno, Nevada, 2007. AIAA-2007-0914.

28. Barth TJ, Jespersen DC. The design and application of upwind schemes on unstructured meshes. Proc. of the 27th AIAA Aerospace Sciences Meeting: Reno, NV, 1989. AIAA-89-0366. 
29. Venkatakrishnan V. Convergence to steady state solutions of the Euler equations on unstructured grids with limiters. Journal of Computational Physics 1995; 118(1):120-130. DOI: 10.1006/jcph.1995.1084.

30. Krivodonova L. Limiters for high-order Discontinuous Galerkin methods. Journal of Computational Physics 2007; 226(1):879-896. DOI: 10.1016/j.jcp.2007.05.011.

31. Koornwinder TH. Askey-Wilson polynomials for root systems of type BC. Contemporary Mathematics 1992; 138:189-204.

32. Mavriplis C. Adaptive mesh strategies for the spectral element method. Computer Methods in Applied Mechanics and Engineering 1994; 116(1-4):77-86. DOI: 10.1016/S0045-7825(94)80010-3.

33. Gottlieb D, Hesthaven JS. Spectral methods for hyperbolic problems. Journal of Computational and Applied Mathematics 2001; 128(1-2):83-131. DOI: 10.1016/S0377-0427(00)00510-0.

34. Sevilla R, Fernandez-Mendez S, Huerta A. NURBS-enhanced finite element method (NEFEM). International Journal for Numerical Methods in Engineering 2008; 76(1):56-83. DOI: 10.1002/nme.2311.

35. Sod GA. survey of several finite difference methods for systems of nonlinear hyperbolic conservation laws. Journal of Computational Physics 1978; 27(1):1-31. DOI: 10.1016/0021-9991(78)90023-2.

36. Burbeau A, Sagaut P, Bruneau CH. A problem-independent limiter for high-order Runge-Kutta Discontinuous Galerkin methods. Journal of Computational Physics 2001; 169(1):111-150. DOI: 10.1006/jcph.2001.6718.

37. Casoni E, Peraire J, Huerta A. Un método de captura de choques basado en las funciones de forma para Galerkin discontinuo en alto orden. to appear in Revista Internacional de Meétodos Numéricos en Ingeniería 2011.

38. Moukalled F, Darwish M. A high-resolution pressure-based algorithm for fluid flow at all speeds. Journal of Computational Physics 2001; 168(1):101-133. DOI: 10.1006/jcph.2000.6683.

39. Luo H, Baum JD, Löhner R. A Hermite WENO-based limiter for Discontinuous Galerkin method on unstructured grids. Journal of Computational Physics 2007; 225(1):686-713. DOI: 10.1016/j.jcp.2006.12.017.

40. Dolejsi V, Feistauer M. A semi-implicit Discontinuous Galerkin finite element method for the numerical solution of inviscid compressible flow. Journal of Computational Physics 2004; 198(2):727-746. DOI: 10.1016/j.jcp.2004.01.023.

41. Anderson JD. Modern Compressible Flow. McGraw Hill Inc.: New York, EUA, 1982.

42. Demirdžić I, Lilek Ž, Perić M. A collocated Finite Volume method for predicting flows at all speeds. International Journal for Numerical Methods in Fluids 1993; 16(12):1029-1050. DOI: 10.1002/fld.1650161202.

43. Hartmann R, Houston P. Adaptive Discontinuous Galerkin finite element methods for the compressible Euler equations. Journal of Computational Physics 2002; 183(2):508-532. DOI: 10.1006/jcph.2002.7206.

44. Emery A. An evaluation of several differencing methods for inviscid fluid flow problems. Journal of Computational Physics 1968; 2(3):306-331. DOI: 10.1016/0021-9991(68)90060-0.

45. Woodward P, Colella P. The numerical-simulation of two-dimensional fluid-flow with strong shocks. Journal of Computational Physics 1984; 54(1):115-173. DOI: 10.1016/0021-9991(84)90142-6.

46. Holden H, Lie K-A, Risebro NH. An unconditionally stable method for the Euler equations. Journal of Computational Physics 1999; 150(1):76-96. DOI: 10.1006/jcph.1998.6164.

47. Xu Z, Liu Y. A conservation constrained Runge-Kutta Discontinous Galerkin method with the improved CFL condition for conservation laws. submitted to SIAM Journal on Scientific Computing 2011. Available at: http: //www.nd.edu/zxu2/publication.html. 